\title{
Ética y liberalismo
}

Witold Jacorzynski

\section{HÉctor Díaz-Polanco, 2007}

Elogio de la diversidad. Globalización, multiculturalismo y etnofagia

Siglo XXI, México, 224 pp.

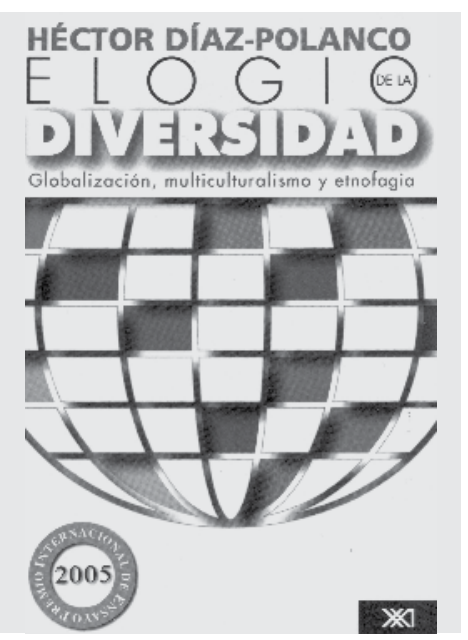

\section{Ethics and Liberalism}

WiTOLD JACORZYNSKI: Centro de Investigaciones y Estudios Superiores en Antropología Social, unidad Golfo, Xalapa, Veracruz, México witusito@yahoo.com.br

Desacatos, núm. 43, septiembre-diciembre 2013, pp. 189-196 libros de Díaz-Polanco es el liberalismo expresado en la famosa teoría de la justicia de John Rawls. Pero aunque DíazPolanco desarrolle el tema del liberalismo rawlsiano en cada uno de sus libros recientes, le agrega un toque nuevo, un argumento adicional que no aparecía en los anteriores. Además, lo hace de manera ingeniosa y asombrosamente versátil. Hay pocos como él que no temen participar en duelos filosóficos, éticos, políticos y antropológicos, siempre con la misma espada, una postura políticamente situada y orientada hacia la liberación de la humanidad de las cadenas del liberalismo. No es posible resumir todos los duelos de DíazPolanco. Me limitaré aquí al tema 
principal del libro: el liberalismo y la liberación de aquél. DíazPolanco escribe:

En resumidas cuentas, con la globalización estamos en una fase en la que el liberalismo es reelaborado para construir una estrategia de "inclusión" de la diferencia (la faceta "magnánima” del imperio), compatible con el capitalismo globalizado. La elaboración más desarrollada de la fundamentación y los principios para esta fase se encuentra, me parece, en la filosofía política de John Rawls y su teoría de la "justicia como imparcialidad" ajustada como "liberalismo político”. La base de esta teoría es universalista y le viene de su sustento kantiano; pero como hemos visto, se ha procurado un ajuste "no metafísico". El efecto del
4) Por tanto, evitemos el liberalismo y elogiemos la diversidad. Veámoslo punto por punto.

\section{¿SI KANT, ENTONCES RAWLS?}

La conexión entre Kant y Rawls no es un cuento del idiota. Kant fue el autor de un sistema ético basado en el principio de la universalidad. Rawls reconoce abiertamente el nexo con el filósofo de Königsberg y afirma que su teoría de la justicia está entendida de manera kantiana, como basada sobre la imparcialidad. Díaz-Polanco cita a Rawls y detecta perspicazmente su intento de construir una teoría de justicia alternativa al utilitarismo, la que sitúa a Rawls en el grupo de los padres de la teoría del contrato social. Este punto es importante, puesto que permite reconstruir los argumentos en favor de la teoría de la justicia desde la perspectiva de Rawls. Rawls cree que en asuntos de justicia la utilidad no se aplica, o como lo observó bien Díaz-Polanco: en Rawls lo justo no es lo mismo que lo bueno. Rawls piensa que lo justo y lo injusto no residen en $l o$ que se elige sino en cómo se elige. La justicia es un fair play, un actuar fair, un juego imparcial; se trata de lo que "se vale" y lo que "no se vale", no tanto de un juego que promueve algún bien, independientemente de lo que "se vale" promover. Esta diferencia es de suma importancia por dos razones: primero, permite distinguir entre las teorías teleológicas - de telos: finy las deontológicas - de deonteis: obligación, deber-. Las primeras, como el utilitarismo, definen nuestras obligaciones en términos de algún fin o bien —felicidad, placer, bienestar o perfección, etc.-, las segundas niegan que nuestras obligaciones sean definibles de esta manera. La ética kantiana es deontológica y formal. Lo único que cuenta en ella es la voluntad que obedece el máximo dictado de la razón práctica. Díaz-Polanco no profundiza en lo que es más difícil de entender en Kant, o sea, el principio de la universalidad. En vez de ello, se concentra en describir la postura de Kant frente al contrato social.

Kant asume que nuestros deberes morales deben ser universales, es decir, que deben obligar a todos en las mismas o parecidas circunstancias. Rawls aplica este criterio a la justicia distributiva. Tenemos que preguntarnos cuáles derechos exigiríamos para nosotros mismos, reconociendo los mismos derechos de otras personas, si tuviéramos que establecer el sistema social y político de novo sin saber nada acerca de nuestras habilidades particulares y gustos: globalización capitalista,
multiculturalismo y etnofagia. 
Los principios de justicia se escogen tras un velo de ignorancia. Esto asegura que los resultados del azar natural o de las contingencias de las circunstancias sociales no darán a nadie ventajas ni desventajas al escoger los principios (Rawls, 1997: 25).

Este método es interesante y se parece a un principio conocido en la ética como "Regla de Oro". No podemos excluir a priori - con base en la condición de la elección desde el velo de ignorancia- que nos tocará una desdicha. Si nos imaginamos que nos toca la desdicha, preferiremos un principio que minimice las desdichas: "Quod tibi fieri non vis alteri ne feceris" - "No hagas al prójimo lo que no quieres que te hagan a ti"-. Pero la "Regla de Oro" no es un invento de Kant ni de Rawls ni de los liberales. Expresa una intuición arraigada en la tradición judeo-cristiana. Rawls cree que este método nos inclina a elegir dos derechos principales:

[1] Cada persona ha de tener un derecho igual al menos más extenso sistema total de libertades básicas compatible con un sistema similar de libertad para todos.

[2] Las desigualdades económicas y sociales han de ser estructuradas de manera que sean: a) para mayor beneficio de los menos aventajados, de acuerdo con un principio de ahorro justo, $\mathrm{y} b$ ) unido a que los cargos y las funciones sean asequibles a todos, en condiciones de justa igualdad de oportunidades (Rawls, 1997: 280).

Aunque Rawls subraya que los principios de justicia han de ser clasificados en un orden lexicográfico y por tanto las libertades básicas sólo pueden ser restringidas a favor de la libertad, el segundo principio tiene el carácter de la estrategia de maximum minimorum - maximin - puesto que propone maximizar las ganancias mínimas. Lo que decidió sobre un gran éxito de la teoría rawlsiana fue su combinación de dos valores, hasta ahora, irreconciliables en teoría liberal: libertad y equidad.

En cuanto Rawls deja muy claro qué es lo que se entiende por universalismo, Díaz-Polanco ofrece al respecto comentarios confusos. Una vez habla sobre el universalismo como opuesto a la diversidad, otra vez lo toma como opuesto al particularismo. Incluso, en alguna parte de su exposición se queja de que:

nada ilustra mejor el abuso del universalismo que el manejo de los derechos humanos para realizar el doble movimiento de imponer una visión particular como universal (pp. 121-122).

Pero ¿qué significa "particular" en este contexto? Un acertijo.
Más adelante, la universalidad de derechos humanos está opuesta a la historicidad de éstos:

Los derechos humanos, de ser prerrogativas históricas construidas por las sociedades, que responden a necesidades concretas de justicia de las agrupaciones humanas, pasan a ser esquemas previos supuestamente fundados en principios ahistóricos, categóricos, absolutos. De ahí les viene su "universalidad" (p. 124).

En otra ocasión se trata de una distinción entre dos versiones de ética apuntadas arriba:

El procedimiento liberal sigue otro camino: definir principios universales (de "justicia"), por ejemplo que excluyan cualquier concepción particular del bien, para subrayar una visión de lo justo que también se pretende universal (p. 125).

En la página siguiente anota:

Su "universalidad", más bien, proviene de la voluntad poderosa de un tipo de sociedad que decide que su visión del mundo debe ser reconocida planetariamente como "la vida buena": la única forma legítima, democrática, de ordenar la sociedad y sus instituciones (p. 216).

En la misma página dice:

[el] derecho a la "intervención humanitaria" comienza a 
figurarse como un nuevo derecho "universal" a la medida de los intereses de los nuevos mandarines de la globalización" (p. 216).

Éstos son algunos ejemplos de un manejo irresponsable de términos como "universal", "universalismo", "universalidad". Entendemos que el autor argumenta contra el principio de la universalidad. No logramos entender, sin embargo, qué es lo que él entiende por este principio y por tanto tampoco contra qué argumenta. Parece que cree que las condiciones culturales distintas imposibilitan el uso del criterio de validez en el sentido de Kant y Rawls. Pero si es así, su argumento es falso. Podemos principio de universalidad aparece como una necesidad inherente al juego de lenguaje moral. En ética no confesamos nuestras penas, intentamos hacer algo más: "no yo y no Pedro sino cada quien en mi lugar y en lugar de Pedro, debe decir la verdad, cumplir con las promesas, no maltratar a los demás, etc.”. Esta formulación filosófica tropieza con dificultades filosóficas, no políticas. Puede ser que el otro no pueda imaginarse en mi lugar o en lugar de Pedro por las situaciones culturalmente diferentes en las cuales estamos yo y Pedro. Nuestras situaciones no se parecen bajo los aspectos relevantes. Pero aun así, la validez universal no se borra: se limita a

la forma de vida o la cultura de los legisladores morales. Kant y su criterio de validez para los principios morales son absolutamente compatibles con la diversidad cultural, puesto que siempre universalizamos algún principio dentro de un cierto universo. Como bien observa Díaz-Polanco, el problema con esta teoría empieza, sobre todo, en el Rawls tardío, quien encuentra problemas filosóficos para expandir la teoría de justicia doméstica más allá de la sociedad liberal. Sería absurdo, no obstante, ver en ello una de las razones en favor de las intervenciones militares planetarias.

Hay aquí algo más. DíazPolanco no puede liberarse de la universalización porque usa el mismo lenguaje que sus supuestos adversarios. Su fuerza argumentativa recurre a la universalidad. ¿No debe obligar planetariamente el principio según el cual un país no debe imponer planetariamente sus propios principios particulares? Sin duda. La confusión más perturbadora de Díaz-Polanco es mezclar dos acepciones de universalidad: una descriptiva y otra normativa. En la primera acepción hablamos sobre un "derecho universal" o una "visión particular como universal" impuesta a todos los países por un país dominante. En otra acepción hablamos sobre una máxima universalizable en el sentido kantiano. Aunque nuestra máxima no se aplica de facto en las circunstancias $X$, debe aplicarse a dichas circunstancias de iure. La diferencia entre las dos acepciones es crucial. Es probable que Estados Unidos, como dice Chomsky, sea el primer terrorista del mundo. Impone pues sus derechos y sistemas políticos en todos los países - los establece "universalmente" en el sentido descriptivo y vago - y sin embargo esta ley no puede tornarse universal puesto que, aunque esto sea discutible, un mundo en el cual cualquier país impusiera militarmente sus leyes sobre otros países sería imposible como ley universal de la naturaleza y como acto de nuestra voluntad. La crítica del universalismo propuesta por Díaz-Polanco es universal.

\section{SI RAWLS, ENTONCES LIBERALISMO}

¿Si liberalismo entonces globalización capitalista...? Las críticas que ofrece Díaz-Polanco a Rawls y al liberalismo son una mezcolanza de comentarios iluminadores y confusos. Empecemos con los primeros. Díaz-Polanco sigue a Hoffman y a Sandel al observar con perspicacia que los árbitros de Rawls, quienes eligen los principios de justicia desde el velo de ignorancia, deben estar ya situados, hecho por el cual no 
pueden ser totalmente

imparciales: "la teoría de la justicia de Rawls presupone una persona ya liberal" (p. 95). Los miembros de las "sociedades jerárquicas", por ejemplo, los musulmanes, no llegan a acordar los mismos principios de justicia. "La razón parece ser simplemente ésta: no llegan a los principios liberales porque no son sujetos liberales" (p. 95). Esta observación parece trivial, pero no lo es. El liberalismo le convence a alguien que ha crecido en las instituciones liberales, quien ya está convencido.

En la mayoría de las sociedades jerárquicas no se eligen los principios de la justicia, se los hereda. Esto no significa que no hay nada que se herede en las sociedades liberales. Nadie puede entender el experimento mental de Rawls si no juega los juegos de lenguaje con "elegir" e "imparcialmente". "Elegir" es un concepto que tiene un lugar especial en las sociedades liberales,

democráticas, capitalistas y consumidoras en las que existe la cultura de "elegir" entre diferentes alternativas, en cuya visión del mundo hay un lugar para tomar las decisiones individuales. Pasa lo mismo con la "imparcialidad". En algunas sociedades jerárquicas la justicia no tiene venda sobre los ojos. Mientras que la ley consuetudinaria de los iroqueses oneida permite que el asesino de una persona sea juzgado por la familia del asesinado a su antojo, varios grupos mayas en Guatemala linchan a los asesinos o los queman vivos. La ley de algunos grupos de esquimales, en cambio, obligaba al asesino de un hombre casado a casarse con la viuda del hombre asesinado. Podemos multiplicar los ejemplos, pero la idea de Díaz-Polanco luce clara: el mundo liberal es distinto del mundo no liberal. El liberal no encuentra puntos de contacto con un no liberal. Otros dos pasos despiertan más dudas. Díaz-Polanco critica a Rawls además de que piensa que es el representante más idóneo del

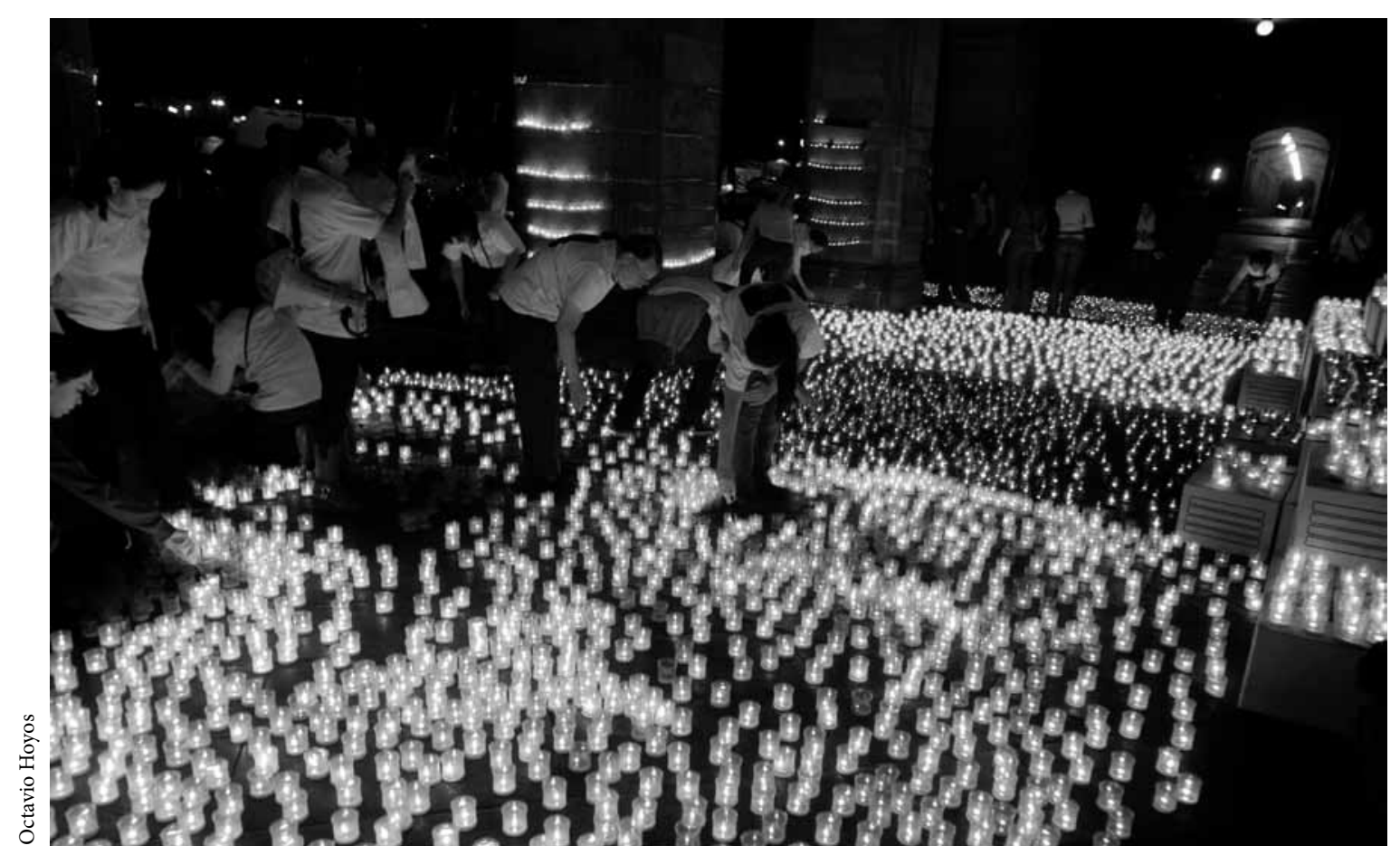

Manifestación de Provida frente al Gobierno del Distrito Federal, 2007. 
liberalismo. Tal postura esencialista hacia el liberalismo produce una confusión total de lo que significa el término "liberalismo" en diferentes contextos. Díaz-Polanco escribe:

el liberalismo predominante (especialmente en sus formulaciones deontológicas más recientes), obtiene un triunfo notable cuando logra encuadrar al menos parte del pensamiento progresista o de izquierda en la lógica de un universalismo que favorece en todo al statu quo capitalista (p. 124).

Esta opinión es falsa, pero útil porque nos revela el posicionamiento ideológico del autor. Existe un pensamiento "progresista" y otro que no lo es. "Progresista" significa aquí que comparte los valores del autor y "no progresista" que no los comparte. Se habla también del liberalismo "predominante", lo que sugiere que hay liberalismo no predominante, dominado o latente. Pero ¿cuáles son los criterios del liberalismo predominante? ¿El número de sus partidarios? ¿El número de las citas de los autores liberales? ¿Su alcance político? Ofrezcamos algunos ejemplos. En cuanto a la justificación, hay por lo menos cuatro liberalismos: el liberalismo teológico de John Locke, el utilitarista de John Stuart Mill, el perfeccionista de Constante y el sociobiológico de Herbert Spencer. En cuanto a su relación con el capitalismo, encontramos a los liberales que creen en el mercado libre sin impuestos y en un Estado minimal - Robert Nozick-, a los liberales que creen en el mercado libre con impuesto progresivo y un Estado de bienestar - John Rawls-y los que rechazan el mercado libre y el capitalismo - Leonard Trelawny Hobhouse y Thomas Hill Green-. Estos últimos son los liberales anticapitalistas. DíazPolanco no menciona este hecho puesto que no encaja con su esquema. Hobhouse y Green creían que el capitalismo ejercía una tiranía moral sobre las personas de tipo medio al impulsarlas a comprar productos que destruyen su salud y autoconfianza (Ryan, 1993: 303). Finalmente, tropezamos con los liberales que enfatizan la importancia de la identidad cultural y los derechos de las minorías étnicas - Will Kymlicka, Joseph Raz-. Tampoco desde la perspectiva libertaria de Robert Nozick hay algún impedimento para que individuos libres puedan rehusarse al capitalismo y aceptar el comunismo.

Díaz-Polanco no logra ver las vicisitudes de diferentes liberalismos porque no aplica el concepto de la semejanza de la familia - aunque, sí, en un sentido retórico e irónico habla sobre una "familia liberal"- - Los liberalismos se parecen entre sí como miembros de la misma familia. Este importante concepto wittgensteiniano permitiría a Díaz-Polanco ahorrar mucha tinta en sus ditirambos contra el liberalismo. Todo depende del aspecto bajo el cual los comparamos y los propósitos de nuestra comparación. El liberalismo descontextualizado no puede ser objeto de ninguna crítica viable. No debemos olvidar que parte de este contexto es su origen. En primer lugar, el liberalismo nace en una lucha revolucionaria contra la tiranía. Liberalismo es un movimiento y una concepción filosófica que se forjó como rebeldía contra diferentes tipos de tiranías y absolutismos, sobre todo religiosos. De esto viene una de sus características compartida por la mayoría de los liberales: el individualismo.

En segundo lugar, el liberalismo no es un sistema ético completo. Se limita a proponer una constitución liberal con su idea principal: lo que no está prohibido, está permitido. La esfera de lo que está permitido es mucho más grande que lo que está prohibido. San Agustín decía: "dilige et quod vis fac" - “ $\mathrm{iAma}$ con consideración y haz lo que quieras!”- - Los liberales dicen: “¡Respeta el derecho de los demás de hacer lo que quieran y haz lo que quieras!" La segunda característica explica en parte los horrores del "neoliberalismo", un fenómeno que no guarda relación lógica con el liberalismo. En los países sobre los cuales versan los 
comentarios de Díaz-Polanco el liberalismo no existe. En lugar de él hay un fenómeno llamado "neoliberalismo", un producto de la incomprensión de lo que es el liberalismo clásico. La perspectiva de sustituir por completo la tradición moral de una sociedad o de grupos étnicos por una constitución liberal tiene que terminar en un desastre, en un vacío axiológico como el que pinta Díaz-Polanco. Pero la tradición moral sin la constitución liberal también termina en un desastre. El liberalismo sin la tradición moral es una arena para los tiburones financieros, hombres de negocios despiadados, políticos corruptos y consumidores egoístas y mediocres. La tradición local sin las garantías de libertad a largo plazo produce muros, prisiones, gulags y campos de concentración característicos de los sistemas totalitarios. ¿Cuál es la solución real? Díaz-Polanco elabora sus propuestas positivas y empieza a argumentar a favor de un principio que él llama "metaprincipio de diversidad".

¿Sería injusto terminar aquí? Después de todo, la incomprensión de un texto puede deberse a las fallas del lector, no a las maniobras del autor. Tal vez lo que propone Díaz-Polanco no es un sueño peligroso sino una alternativa política real, la de la "izquierda" real. Efectivamente, el autor ofrece varios ejemplos interesantes. Entre ellos, el neozapatismo y el chavismo. El primer fenómeno es demasiado complejo para discutirlo aquí. No se deja clasificar tan fácil como lo hace Díaz-Polanco: no se deja encajonar como "profunda reforma" ni como "antipoder" (p. 208). Pero el ejemplo del chavismo es inquietante (p. 188). Díaz-Polanco argumenta inesperadamente que los ataques lanzados a Chávez por los intelectuales que representan las sociedades democráticoliberales son injustificados. Chávez "fue elegido" en elecciones democráticas. Bien puede ser que el lector no entienda al autor, pero más probable es que el autor se burle del lector. Primero, si el gobierno de Chávez es justo porque fue elegido democráticamente, el gobierno de Hitler también lo fue porque fue elegido democráticamente en 1933. Segundo, parece que DíazPolanco olvidó en la página 188 que en las páginas anteriores criticaba la universalidad de los ideales democráticos y liberales. Parece que el principio de una coherencia lógica mínima también es un principio que debe ser negociado en una nueva feliz academia.

Y más adelante el autor admite que el liberalismo universalista, en oposición a la izquierda progresista, es "etnófago". Entendemos que significa "el antropófago". El Cíclope, el hijo de Poseidón, tragó a varios compañeros de Odiseo en el poema de Homero. Coprofagia es otro ejemplo desagradable, una señal de la deficiencia de las vitaminas en el perro. Pero ¿etnofagia? ¿Qué es lo que come el etnófago? Díaz-Polanco debe ofrecernos en este momento una concepción inteligible de las etnias, pero no lo hace. El término "etnia" carece del referente, de la misma manera que el término "liberalismo". Pero si carece del referente ¿cómo puede ser entendida esta noción?

No lo podemos negar. El juego del lenguaje en el cual se juega con grupos étnicos pertenece a un cierto dominio del lenguaje: la jurisdicción internacional, la política, el funcionamiento de las instituciones que se ocupan de los derechos indígenas y la lucha por poder en éstas. Este dominio del lenguaje se relaciona estrictamente con lo que Marx llamaba "la ideología" o la "falsa conciencia”. Entendemos qué significa "grupo étnico" si entendemos cómo, dónde y en qué circunstancias circula y está usado el término "grupo étnico". ¿Quién gana y quién pierde al usarlo? ¿Los intereses de quiénes realiza éste u otro empleo de esta palabra mágica? Bajo esta perspectiva, sería absurdo hablar de la etnofagia. En términos más posmodernos, los "grupos étnicos" no son "comida" para los etnófagos sino una construcción de los políticos, antropólogos y abogados en cuyo 
interés está hablar sobre la etnofagia y los etnófagos. Esta construcción, como otras tantas, deben ser deconstruidas en el proceso de un análisis conceptual serio. Esto no significa que los grupos étnicos no existan o que sean un cuento del idiota, sino que tan sólo necesitamos un contexto, un juego de lenguaje apropiado para poder desentrañar las reglas de su uso. En conclusión, el trabajo de
Díaz-Polanco es un reto tan interesante como fallido. Es interesante porque pretende comentar críticamente una cierta tradición arraigada en nuestro pensamiento político como baobab. Este baobab, como en el planeta del Principito, ha logrado penetrar nuestras tradiciones políticas hasta el fondo - libertad, democracia, derechos, elecciones, fraudes, igualdad, justicia, etc.-. Y es fallido porque no encuentra en punto arquimediano sobre el cual pueda fundarse su crítica.

\section{BIBLIOGRAFÍA}

Rawls, John, 1997, Teoría de la justicia, Fondo de Cultura Económica, México.

Ryan, Alan, 1993, "Liberalism”, en Robert E. Goodin y Philip Pettit, A Companion to Contemporary Political Philosophy, Blackwell, Cambridge. 